Check for updates

Cite this: RSC Adv., 2018, 8, 35094

\title{
A monolithic anti-freezing hydro/organo Janus actuator with sensitivity to the polarity of solvents $\uparrow$
}

\begin{abstract}
Chao Fang, Kaixiang Yang, Qiang Zhou, Kang Peng and Haiyang Yang (D) *
Hydrogels with bilayer structures, known as actuators, have attracted increasing attention in recent years due to their sophisticated intellectual functions such as pine-cone-like bending and pod-like twisting in response to temperature, light, $\mathrm{pH}$, special solvents etc. Herein, we synthesized a monolithic hydro/ organic actuator by a direct one-step interfacial-incompatible polymerization of hydrophilic and hydrophobic vinyl macromolecular monomers, PEGMA and PPGMA, which were dissolved in water and ethyl acetate solutions, respectively. We found that the hydro/organo actuator exhibited directional, bidirectional, and site-specific bending behaviour under a single stimulus. In water and non-polar organic solvents, the hydro/organo actuator exhibited opposite bending behaviour as expected. However, in organic solvents, the hydro/organo actuator also exhibited quite different bending behaviour, depending on the polarity of the organic solvent. The hydro/organo actuator even experienced a dual responsive action in response to a single stimulus. We also found that such a hydro/ organo actuator could be used effectively at low temperatures. The mechanism and potential applications of hydro/organo actuators based on PEGMA- $b$-PPGMA were also discussed and provided.
\end{abstract}

Received 10th August 2018 Accepted 27th September 2018

DOI: 10.1039/c8ra06719h

rsc.li/rsc-advances a pretreatment process such as dialysis, which was quite timeconsuming. In order to solve the above problems, Jiang et al. ${ }^{29}$ obtained hydro/organo Janus actuators by using a special interfacial polymerization method based on the characteristics of hydrophilic monomer aqueous solutions and hydrophobic monomer organic solutions. The resulting amphiphilic block copolymer with different hydrophilic/hydrophobic motions can act as an intelligent sensor element for solvent leak detection. The above Janus actuator can be used in water as well as in organic solvents. However, it cannot be used at low temperatures, and has the same bending direction in different organic solvents. In other words, the bending direction is not selective for polarity of the organic solvent. There are no reports of actuator materials that can be used at low temperatures that bend in relation to solvent polarity.

Hydrogels have an obvious disadvantage. If the temperature is lowered below $0{ }^{\circ} \mathrm{C}$, hydrogels freeze and become rigid and brittle. ${ }^{30}$ Therefore, in all actuators which consist of hydrogels, the hydrogels lose their desirable properties once the temperature decreases below the freezing point of water, severely limiting their use in this temperature range. Enlarging the temperature range of applications of actuators is an urgent problem to be solved.

Polyethylene glycol (PEG) is a synthetic polymer which is a linear or branched, nontoxic, and biocompatible macromolecule that can be soluble in water and various organic solvents. ${ }^{31}$ Amphiphilic gels - another area of usage of PEG - are three-dimensional networks which contain both hydrophilic and hydrophobic segments that can absorb water and organic
CAS Key Laboratory of Soft Matter Chemistry, School of Chemistry and Materials Science, University of Science and Technology of China, Hefei 230026, P. R. China. E-mail: yhy@ustc.edu.cn

$\dagger$ Electronic supplementary information (ESI) available. See DOI: 10.1039/c8ra06719h 
solvents. ${ }^{31-33}$ In addition to the high water absorption properties of these PEG-based materials, recently studied PEGDA polymer materials have a high absorption capacity in various polar solvents, ${ }^{34-36}$ but do not absorb non-polar solvents. ${ }^{34}$

Polypropylene glycol (PPG), which is usually produced by the ring opening polymerization of propylene oxide, is a polymer with alternating hydrophobic and hydrophilic groups in molecular chains. PPG displays some degree of hydrophobicity due to the additional methyl group in each repeating unit. It is insoluble in water, especially when the molecular weight is greater than $740 \mathrm{~g} \mathrm{~mol}^{-1}$. PPG polymers can demonstrate highly hydrophobic properties at higher molecular weights. ${ }^{37-41}$ The PPG structure has good polarity and solubility as well as good stability and hydrolysis resistance as reported. ${ }^{42}$

Here, due to incompatibility between the hydrophilic vinyl monomer PEGMA (polyethylene glycol methyl ether methacrylate) and the hydrophobic high molecular weight monomer PPGMA (polypropylene glycol monobutyl ether acrylate), we macroscopically prepared hydro/organo Janus copolymer strips by interfacial polymerization of PEGMA and PPGMA (Fig. 1). These hydro/organo Janus copolymer strips showed a multiresponsive shape transition (Fig. 3). The binary synergy of the hydrogel and organogel networks allows the Janus copolymer strips to have unidirectional and specific bending in aqueous solutions and various organic solvents. All of these shape transitions are due to the combined effect of different expansion coefficients between the hydrogel network and the organogel network in the copolymer strips. In addition, due to the hydration of PEGMA, the PEGMA hydrogel does not freeze at low temperatures, making PEGMA- $b$-PPGMA Janus copolymers usable at low temperatures. We therefore first produced an actuator which responds to various organic solvents, but this Janus copolymer also has the unique characteristic of solvent polarity response. Apart from the general actuator performance, both PEGMA and PPGMA gels can be used in many organic

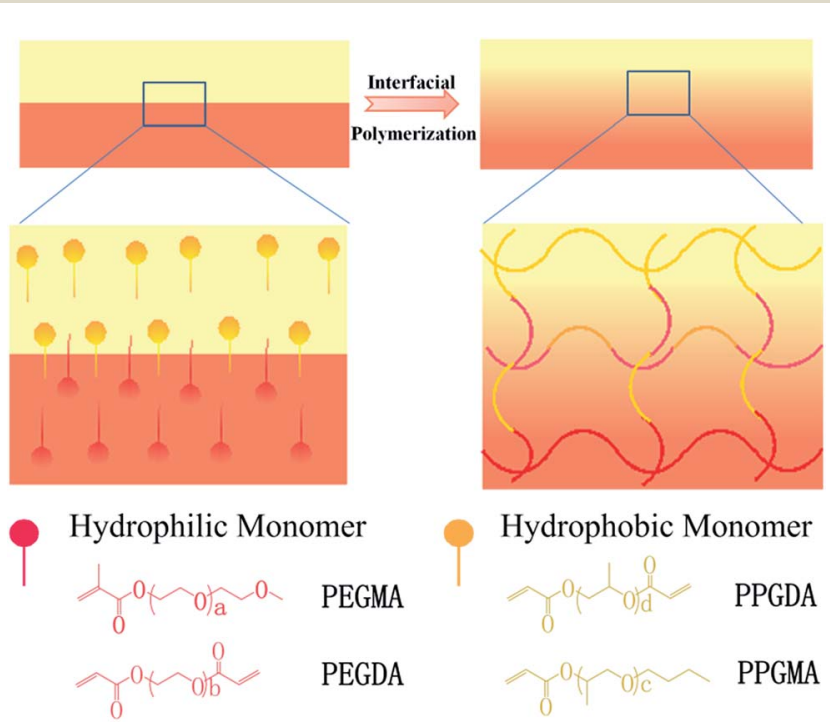

Fig. 1 Design of the shape-shifting hydro/organo Janus copolymer strips. The Janus strip was synthesized via an interfacial polymerization of hydrophobic and hydrophilic monomer solutions.

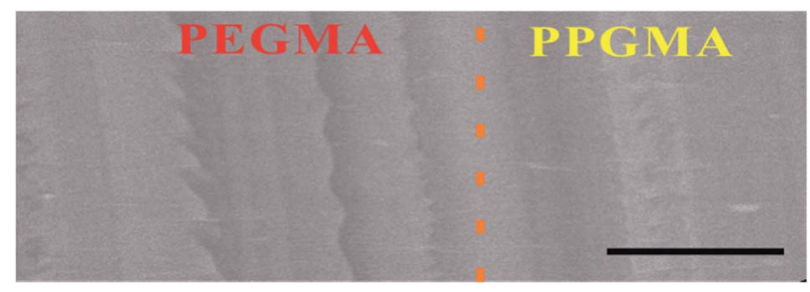

Fig. 2 SEM images of the cross-section of the Janus copolymer; the scale bar is $10 \mu \mathrm{m}$.

solvents, so that when a part of the Janus copolymer is immersed in a solvent, it can be bent. When completely immersed, the Janus strip has a second response which is gradual flattening or even reverse bending. In addition, the synergy between the hydrogel network and the organogel network allows the Janus copolymer strips to function as an intelligent sensor element for solvent leak detection. With its dual response, alarming and post-disposal of solvent leaks can be performed spontaneously. This provides a potential new idea in the fields of industrial application (alarm and postprocessing of fractionation tower fractions) and medical treatment (lung fluid detection and treatment).

\section{Experimental}

\subsection{Materials}

Acrylic acid (AA), polypropylene glycol 2000 (PPG, $\left.M_{\mathrm{n}}=2000\right), p$ toluenesulfonic acid ( $p$-TsOH), hydroquinone, sodium bicarbonate $\left(\mathrm{Na}_{2} \mathrm{CO}_{3}\right)$, polypropylene glycol monobutyl ether 2500 (PPGM, $M_{\mathrm{n}}=2500$ ), petroleum ether (PE), ethanol (EtOH), ethyl acetate (EA), toluene (TL), tetrahydrofuran (THF), acetone (CP), $\mathrm{N}, \mathrm{N}$-dimethylformamide (DMF) and benzene were purchased from Sinopharm Chemical Reagent Co. Ltd. Poly(ethylene glycol) methyl ether methacrylate 500 (PEGMA, $\left.M_{\mathrm{n}}=500\right)$, poly (ethylene glycol) diacrylate 700 (PEGDA, $M_{\mathrm{n}}=700$ ), 1-hydroxycyclohexyl phenyl ketone and 2-hydroxy-4'-(2-hydroxyethoxy)-2methyl benzophenone were purchased from Sigma-Aldrich. All chemicals used were of reagent grade without further purification.

\subsection{Synthesis of polypropylene glycol diacrylate (PPGDA) and polypropylene glycol monobutyl ether acrylate (PPGMA) $)^{42,43}$}

In a $500 \mathrm{~mL}$ three-necked flask, $0.025 \mathrm{~mol}$ PPG, $0.035 \mathrm{~mol}$ acrylic acid, $50 \mathrm{mg}$ hydroquinone as an inhibitor and $200 \mathrm{~mL}$ benzene as the solvent were added, followed by refluxing for 2 hours. Subsequently, $0.0029 \mathrm{~mol} p$-toluenesulfonic acid was added and the mixture was refluxed for 9 hours. Then, sodium carbonate was added to stop the reaction, and was kept stirring for 2 hours at $40{ }^{\circ} \mathrm{C}$. The reaction mixture was filtered and the remaining solvent was evaporated on a rotary evaporator to gain the product (yield: 94.18\%). The synthesis of PPGMA (yield: $93.58 \%$ ) was carried out by the same procedure as that for PPGDA (ESI Scheme S1†). 
a

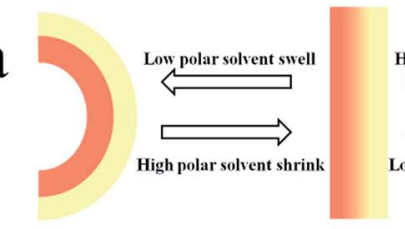

Hydrogel Inwards

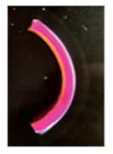

Original State
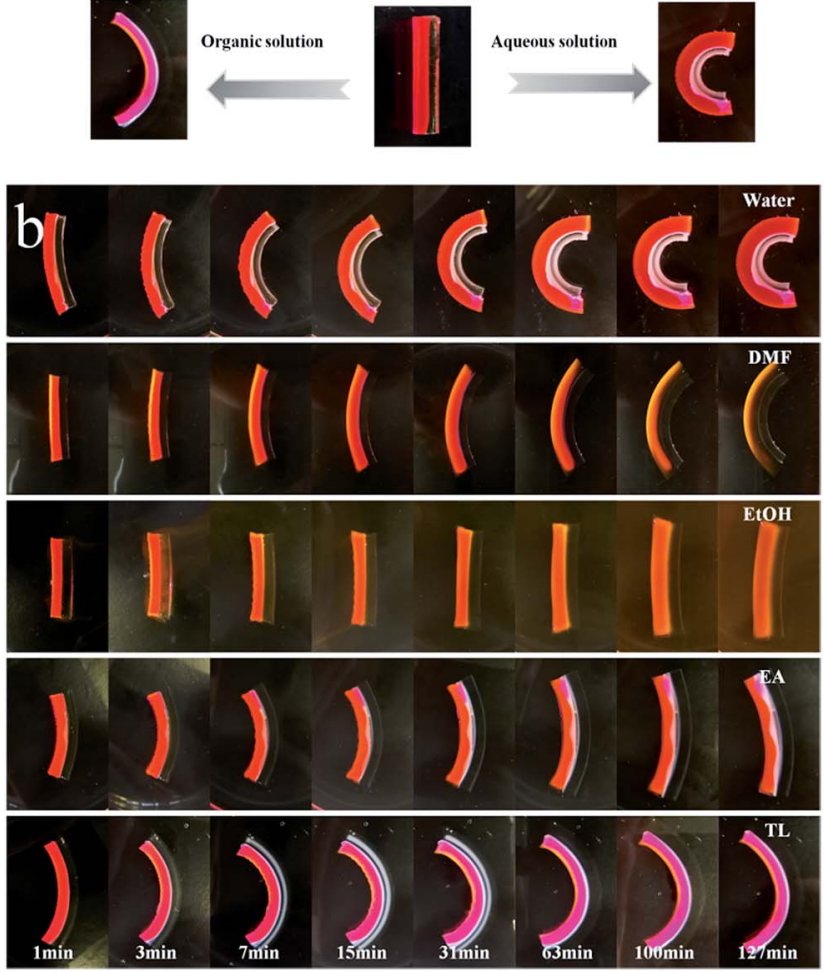

Fig. 3 (a) Bidirectional bending under the binary cooperative effect of the hydrogel network and the organogel network. Immersed in high polarity solvents (aqueous solution), the hydrogel network of the Janus strip had a greater swelling rate than the organogel, leading to bending of the Janus strip with the organogel network inwards. The Janus strip bends with the hydrogel network inwards when immersed in low polarity solvents (toluene), due to the swelling of the organogel network. The obtained PEGMA hydrogel networks were dyed tawny with rhodamine. (b) Photographs of the bending processes.

\subsection{Preparation of the PEGMA- $b$-PPGMA Janus actuator}

The reactive aqueous solution was composed of $5 \mathrm{~mol} \mathrm{~L}^{-1}$ monomer PEGMA500, PEGDA700 (1:200 molar ratio of monomer) as the crosslinker, and 2-hydroxy-4'-(2-hydroxyethoxy)benzophenone $\left(10 \mathrm{mg} \mathrm{mL} \mathrm{mL}^{-1}\right.$ reaction solution) as the photoinitiator. The reactive organic solution consisted of $5 \mathrm{~mol} \mathrm{~L}^{-1}$ monomer PPGMA2500, PPGDA2000 (1 : 200 molar ratio of monomers) as the crosslinker, and 1-hydroxycyclohexyl phenyl ketone $\left(20 \mathrm{mg} \mathrm{mL}{ }^{-1}\right.$ reaction solution) as the photoinitiator. Then, a certain amount of the hydrophilic reactive solution and the organic reactive solution were successively added to a mold (the mold consisted of two glass slides sandwiching a certain thickness of rubber ring). After brief mixing, the reaction was carried out under a $365 \mathrm{~nm}$ UV lamp for 4 hours. Finally the network structure of the PPGMA- $b$-PEGMA Janus copolymer was obtained (ESI Scheme S2†).

\subsection{Preparation of the control gels}

To analyze the performance of the PEGMA- $b$-PPGMA Janus strips, PEGMA gels and PPGMA gels were prepared separately in the mold to study their respective properties.

Preparation of PEGMA hydrogels. The reactive aqueous solution consisted of $5 \mathrm{~mol} \mathrm{~L}{ }^{-1}$ of monomer PEGMA, with PEGDA (1 : 200 molar ratio of monomer) as the crosslinker, and 2-hydroxy-4'-(2-hydroxyethoxy) benzophenone $\left(10 \mathrm{mg} \mathrm{mL}^{-1}\right)$ as the photoinitiator. Next, the gel was photopolymerized under UV light (365 $\mathrm{nm}$ ) irradiation for $4 \mathrm{~h}$.

Preparation of PPGMA Organogels. The reactive organic solution consisted of $5 \mathrm{~mol} \mathrm{~L}{ }^{-1}$ of monomer PPGMA, with PPGDA (1:200 of monomer molar ratio) as the crosslinker and 1-hydroxycyclohexyl phenyl ketone $\left(20 \mathrm{mg} \mathrm{mL}^{-1}\right)$ as the photoinitiator. Next, the gel was photopolymerized under UV light $(365 \mathrm{~nm})$ irradiation for $4 \mathrm{~h}$.

\subsection{Characterization of the Janus actuator}

SEM observation. The surface morphology of the bilayer Janus copolymer was observed by scanning electron microscopy (SEM, JSM-6700F). The prepared Janus copolymer was immersed in deionized water for $24 \mathrm{~h}$ and then freeze-dried for $24 \mathrm{~h}$ to remove water thoroughly. Before the observation, the freeze-dried gel was carefully sliced and sputtered with gold.

Thermal Characterization. The samples were characterized using a differential scanning calorimeter (TA Instruments, DSC Q2000) with a refrigerated cooling system (TA Instruments, RCS90). The samples were contained in hermetically sealed aluminum pans (TA Instruments, Tzero Aluminium Hermetic Pan) for testing, with an empty pan used as an inert reference. The DSC was operated under a $40 \mu \mathrm{L} \mathrm{min}{ }^{-1}$ nitrogen flow rate and data were captured at a rate of $1 \mathrm{~Hz}$. Samples were first equilibrated at $25{ }^{\circ} \mathrm{C}$ and then cooled at a rate of $5{ }^{\circ} \mathrm{C} \mathrm{min}{ }^{-1}$ to $-90{ }^{\circ} \mathrm{C}$. After an isothermal period of at least $90 \mathrm{~min}$, the samples were heated up at $5{ }^{\circ} \mathrm{C} \mathrm{min}^{-1}$ to the initial equilibration temperature $\left(25^{\circ} \mathrm{C}\right)$.

\subsection{SR, SRR and $\kappa$ measurements}

The prepared PEGMA hydrogels and PPGMA organogels were immersed in different solvents respectively, and the swelling quality was measured at different times. The swelling rate (SR) was calculated as follows: ${ }^{44,45}$

$$
\mathrm{SR}=\frac{W_{\mathrm{s}}}{W_{\mathrm{d}}} \times 100 \%
$$

$W_{\mathrm{d}}$ is the weight of the original gel, and $W_{\mathrm{s}}$ is weight of the swollen gel.

The rate of swelling rate (SRR) was defined as follows:

$$
\mathrm{SRR}=\frac{\mathrm{SR}(\text { PEGMA })}{\mathrm{SR}(\text { PPGMA })}
$$

The Janus strip was cut into rectangular strips and immersed in different solvents to observe their curvatures. The curvature was calculated as follows: ${ }^{45,46}$ : 


$$
\kappa=\frac{\theta \pi}{180 \times L}
$$

where $\kappa$ is the curvature and $\theta$ is the bending angle, as shown in eqn (3), $L$ is the free length of the Janus strip, so $\kappa$ is proportional to $\theta / L$. An illustration of $\theta$ is shown in ESI Scheme $\mathrm{S} 3 \dagger$ and Fig. 4c.

\section{Results and discussion}

\subsection{Swelling of actuators in various solvents}

The successful preparation of PPGMA and PPGDA was confirmed by their FTIR spectra (Fig. S1 $\dagger$ ) and ${ }^{1} \mathrm{H}$ NMR (Fig. S2 $\dagger$ ).

The hydro/organo Janus copolymer strip was polymerized from a bilayer solution of PEGMA and PPGMA, which were the hydrophilic monomer and hydrophobic monomer, respectively (Fig. 1a). Due to their different densities, the aqueous solution with the larger density was added to the mold to spread over the surface. Then, the organic solution was added to form the upper layer. After a short standing time, polymerization was initiated by UV light and interfacial polymerization of the hydrophobic monomers and hydrophilic monomers occurred at the oil/water interface (ESI Scheme S2 $\dagger$ ). A monolithic Janus copolymer was obtained, which provided the Janus strip with both properties due to the presence of the hydrophilic network and the hydrophobic network.

The internal morphology of the assembled bilayer Janus copolymer was observed using a scanning electron microscope (SEM) (Fig. 2). The boundary of the two layers was distinct, but in tight conjunction. No obvious cracks were observed from the scanning electron microscopic images (Fig. 2) and the existing structural gradient confirmed the Janus properties of the strip. The binary structure of the hydrogel and organogel network structures gave the strip responsiveness in different environments. In order to explore the mechanism of their shape transition, we quantitatively studied the Janus strip's bending behaviours by measuring the ratio of swelling ratio (SRR) (eqn (2)) and the curvature $\kappa$ (eqn (3)).

The PEGMA network of the Janus strip swelled in the aqueous solution, resulting in unidirectional bending towards the PPGMA. The swelling of the PPGMA network caused the Janus strip to bend towards the PEGMA network (Fig. 3a, S7 and ESI Movie S1 $\dagger$ ). The PPGMA organogel was found to swell in organic solvents such as petroleum ether, toluene, ethyl acetate, ethanol, and DMF etc. (Fig. S3†). However, we found that except for essentially not being swollen in petroleum, PEGMA could swell in most organic solvents (Fig. S3†). Then we investigated the swelling behavior of PPGMA and PEGMA in solvents of different polarities. We found that the SRR (eqn (2), Fig. 4a and $\mathrm{S} 4 \dagger$ ) grew gradually with an increase in solvent polarity. When the polarity was lower than 4.3 , with increasing polarity, the SRR tended to 1 . Similarly, when the polarity was higher than 4.3, with decreasing polarity the SRR tended to 1 . The polarity of 4.3 was the demarcation point. At the demarcation point, the swelling properties of the PEGMA network and the PPGMA network were basically the same. Macroscopically, the Janus strips were not bent in these solvents at short times.
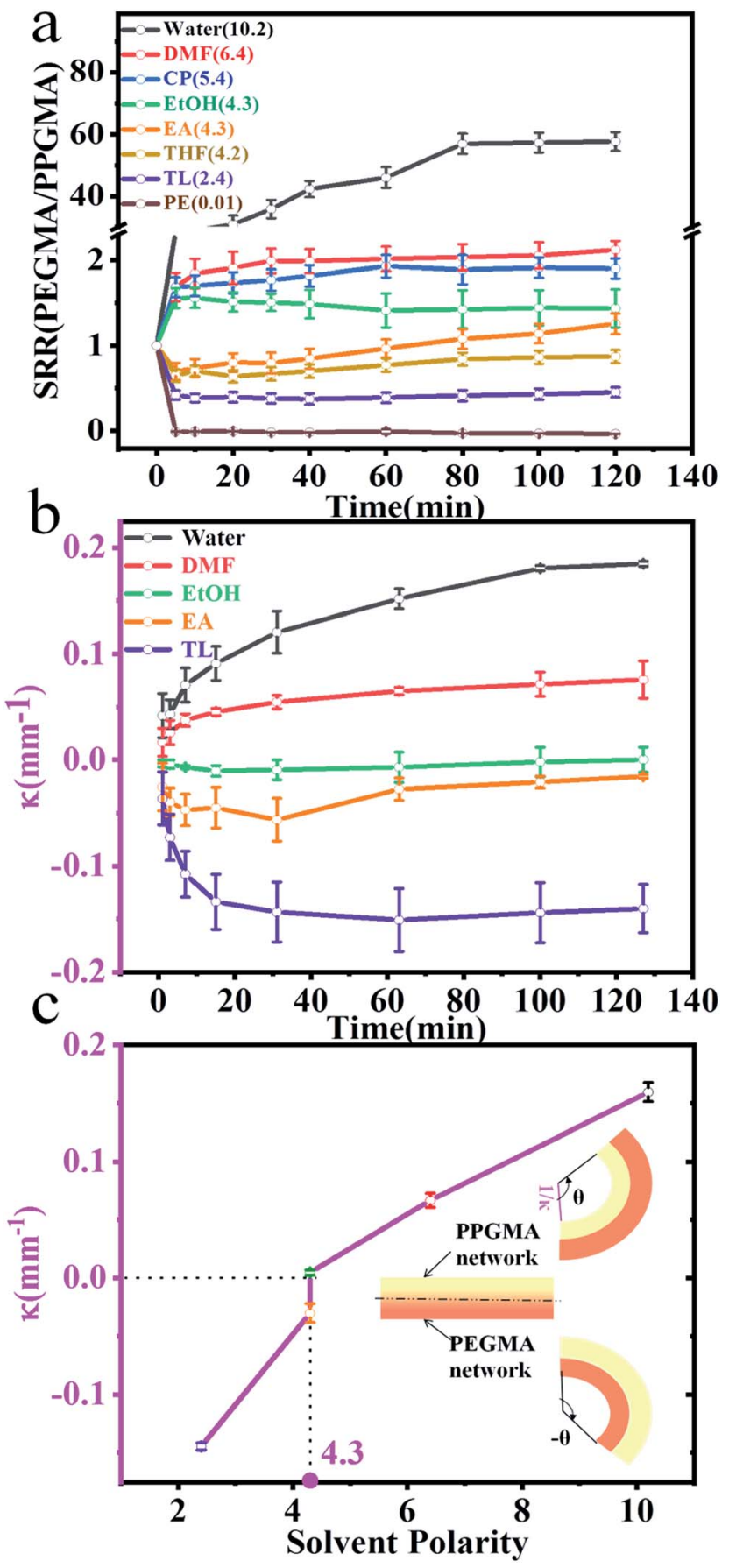

Fig. 4 (a) Comparison of the rate of swelling rate (SRR) in different media. (b) The corresponding curvature of the PEGMA-b-PPGMA Janus strip actuators during the dynamic process. (c) Comparison between the curvature of the Janus copolymer consisting of the PEGMA network and PPGMA in various polar solvents. A negative angle represents bending downward toward the PEGMA network of the Janus strip, and a positive angle represents bending upward toward the PPGMA network of the Janus strip.

\subsection{Effect of polarity of solvents on the actuator's bending} behaviors

The hydro/organo actuator should, being more intelligent than previous actuators, bend itself differently in different organic 
solvents, depending on the polarity of solvent. In order to verify this view, we then prepared an actuator where v(PEGMA)/ $\mathrm{v}($ PPGMA $)=1$. The PEGMA- $b$-PPGMA Janus strip actuators were placed in five solvents: water, DMF, ethanol, ethyl acetate, and toluene, to observe the bending (Fig. 3b). For the PEGMA- $b$ PPGMA Janus strip actuator, the trend of the response curvature (Fig. 4b) was in line with its SRR (Fig. 4a) due to the binary synergistic swelling efficiency of the PEGMA network and the PPGMA network structure in these five solvents. Fig. 4c displays the bending angles of the Janus copolymer as a function of solvent polarity, where a negative angle represents bending toward the PEGMA network of the Janus strip, and a positive angle represents bending toward the PPGMA network of the Janus strip (ESI Scheme S3†). The Janus strip bent toward the PEGMA network in lower polarity solvents, whereas they bent toward the PPGMA network under higher polarity solvents. The cutoff point of the bending direction of the Janus copolymer is at a polarity of 4.3 (Fig. 4c).

We then made a "+" Janus strip, which bent downwards when immersed in toluene. However, when immersed in water, it flattened and eventually turned upwards (ESI Fig. S5 and Movie S2 $\dagger$ ). On account of the incompatibility between polar and non-polar solvents, the replacement of different solvents will inevitably take some time, but we can reduce the response time by decreasing the thickness of the samples (Fig. S6†).

\subsection{Bidirectional bending in one solvent and specific-site bending in the oil/water binary system}

A typical bilayer actuator consists of an active layer and a passive layer, and can only respond once to external stimuli (Fig. 5a(iiii)). The PEGMA- $b$-PPGMA Janus copolymer actuator we synthesized not only has general actuator characteristics but also has a dual response (Fig. 5a(i-vi) and ESI Movie S4 $\dagger$ ). Therefore, bidirectional bending was achieved by the PAA- $b$ PBMA Janus strip in one solvent, because the PEGMA network and the PPGMA network can swell in many solvents and therefore these are all active layers (Fig. S3†). When only one layer is immersed in an organic solvent, due to the swelling of the layer, the Janus copolymer starts to bend upwards (Fig. $5 \mathrm{a}(\mathrm{i}-$ iii)). When the solvent completely submerges the Janus copolymer, the degree of bending of the copolymer decreases or it even bends downward due to the swelling of the second layer (Fig. 5a(iv-vi)). To verify this point of view, we placed the Janus copolymer in a glass Petri dish, where the lower layer is the PEGMA network and the upper layer is the PPGMA network (Fig. 5b(i)). An organic solvent (ethyl acetate) was added with the height of the solvent kept under half that of the strip (only the PEGMA network was immersed in the solvent) and the Janus strip bent upward due to the swelling of the PEGMA network (Fig. 5b(iii)). Then organic solvent (ethyl acetate) was added continuously so the Janus strip was completely immersed in the organic solvent (Fig. 5b(iv)). Due to the synergistic effect of the swelling of the PPGMA network and the PEGMA network, the Janus strip was gradually bent downward (Fig. 5b(vi)). As a result, under the binary cooperation between the hydrogel and organogel networks, the bending direction of the PEGMA- $b$ -
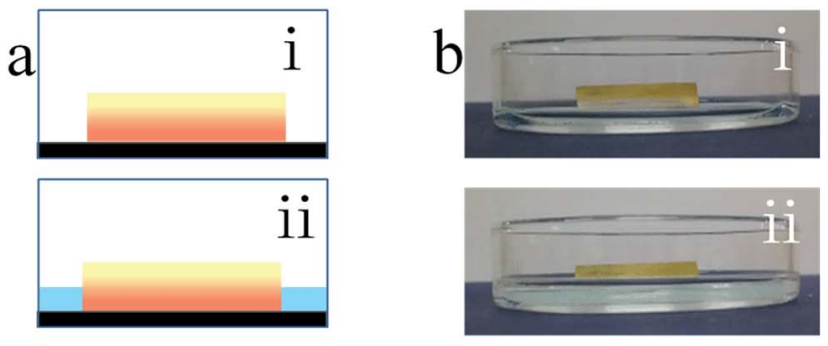

ij
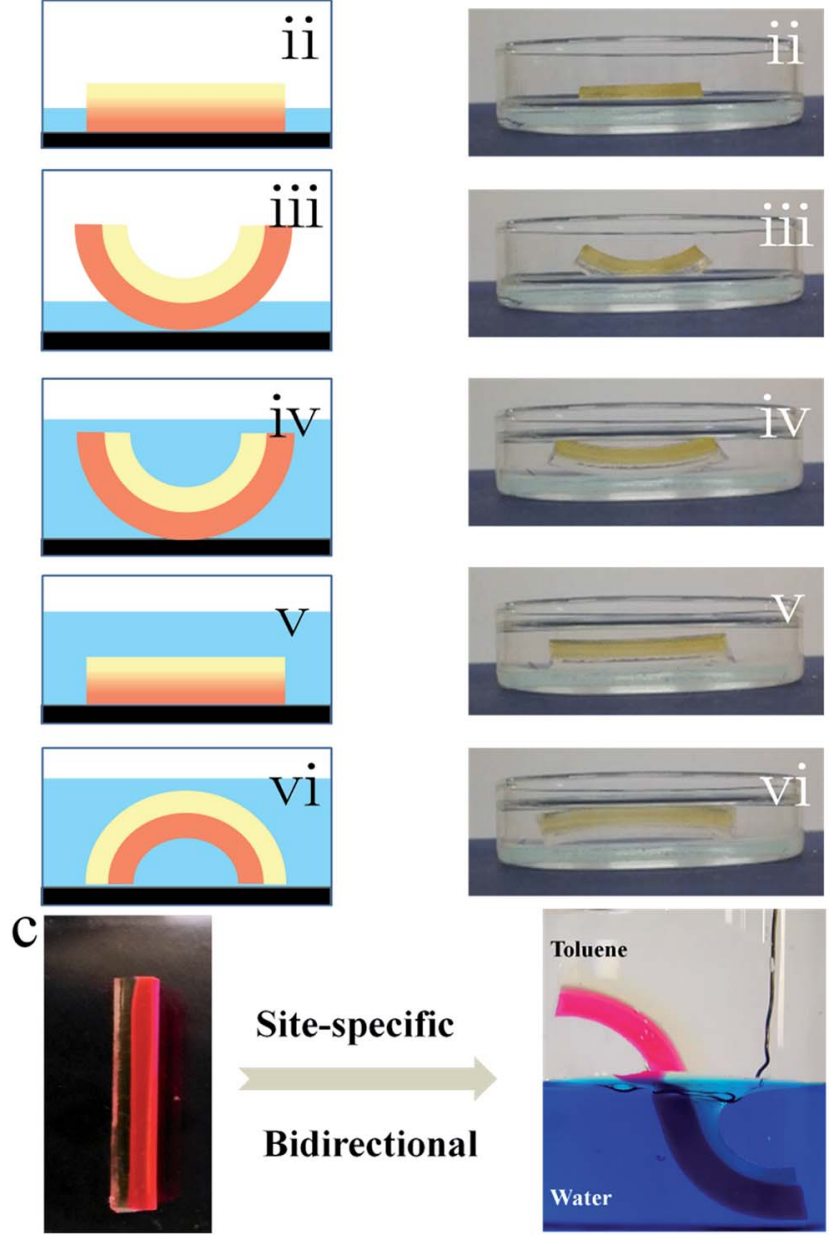

$\mathrm{Vi}$
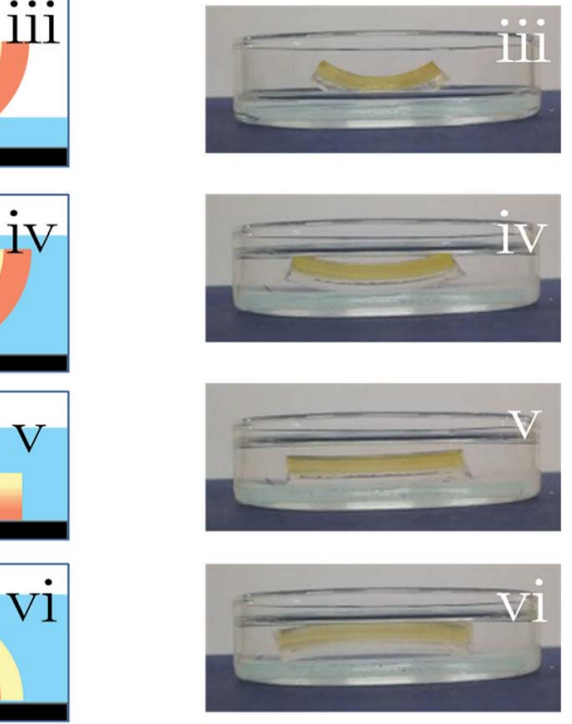

Fig. 5 PEGMA- $b$-PPGMA Janus strip. (a) A schematic diagram of the dual response, (b) a photo of the dual response (with ethyl acetate as the solvent) and (c) the site-specific bending of the Janus strip. When immersed in aqueous solution/organic solvent systems, the Janus strips displayed bidirectional bending with the water/oil interface as the cut-off point. In a TL (toluene)/water system, the upper part bent with the PEGMA inwards, whereas the bottom part bent with the PPGMA inwards. The water was dyed blue with methylene blue.

PPGMA Janus strip could be regulated in one solvent just by adjusting the height of the strip immersed in the solvent.

The specific-site bending of the PEGMA- $b$-PPGMA Janus strip in an oil/water binary system was also successfully achieved. The Janus strip immersed in the aqueous solution/organic solvent showed a specific binary bending at the cutoff point (Fig. 5c). In the above toluene solution, it bent to the left, and in the below aqueous solution it bent to the right.

\subsection{Bending behaviors at low temperatures}

We stored the PPGMA organogel, the pure PAA hydrogel, and the pure PEGMA hydrogel at $-20{ }^{\circ} \mathrm{C}$ for 24 hours. The pure PPGMA organogel was found to have good flexibility at low 
temperatures (Fig. 6a). The pure PAA hydrogel was a softer gel at room temperature, but the hydrogel lost its flexibility at $-20{ }^{\circ} \mathrm{C}$ due to the freezing of water (Fig. 6a). However, pure PEGMA hydrogels were still flexible at $-20{ }^{\circ} \mathrm{C}$ (Fig. 6a) because the hydrogen-bonded hydration between the PEGMA network and water in the PEGMA hydrogel made water molecules anchor firmly in the polymer network, ${ }^{47-49}$ and the PEGMA hydrogel was therefore frost-free. Peaks in the DSC curve ${ }^{30,50,51}$ indicate that the PEGMA hydrogel can be cooled to temperatures as low as $-62{ }^{\circ} \mathrm{C}$ without freezing (Fig. 7). Subsequently, we placed the PEGMA- $b$-PPGMA Janus Copolymer strips, which had been stored at $-20{ }^{\circ} \mathrm{C}$ for 24 hours, into an organic solvent (DMF) at $-20{ }^{\circ} \mathrm{C}$ and they could still bend in organic solvents at low temperature. This indicated (Fig. $6 \mathrm{~b}$ and S8 $\dagger$ ) that these Janus Copolymer strips can still be used at low temperatures.

\subsection{Potential application}

Soft actuators with variable shapes and extensive adaptive abilities are required for applications in many fields such as smart sensors, circuit switches, and soft grippers. The Janus strips of PEGMA- $b$-PPGMA are particularly responsive in different solvents and thus serve as detectors for solvent leaks. We could clearly distinguish between two solvents with a similar polarity at around 4.3 through the bending direction. To prove this, we designed a simple Janus strip with PEGMA- $b$ PPGMA as the sensing element (Fig. 8). As a high polarity solvent was added, the Janus strip began to bend toward the PPGMA network structure and touched the right plate, causing the right circuit to switch on, and the right light was turned on. Conversely, when the low polarity solvent leaked, the Janus strip

\section{a}
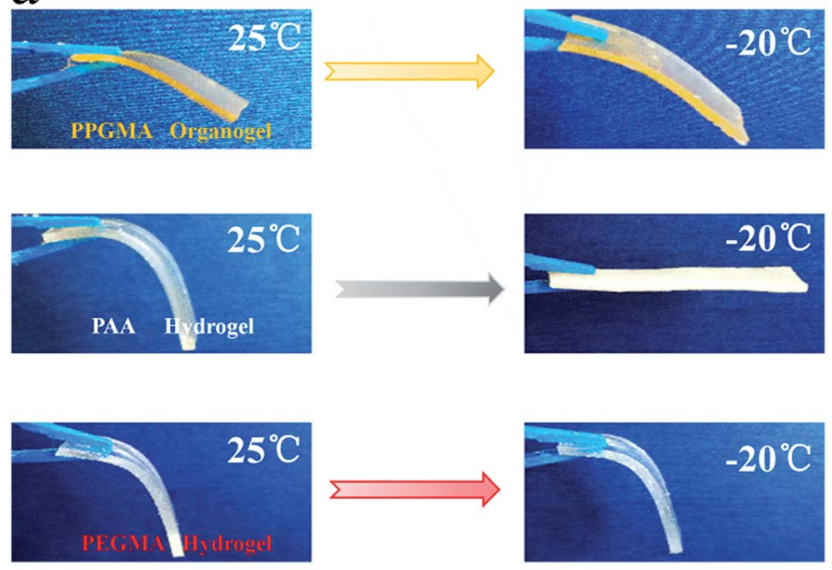

b
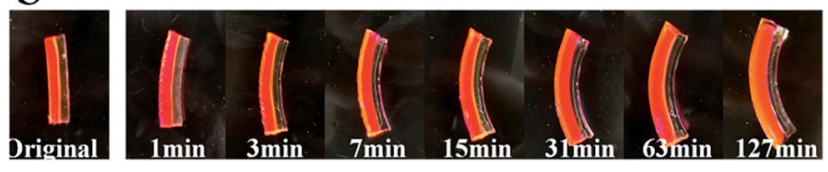

Fig. 6 (a) Photo of the PPGMA organogel, the PAA hydrogel, and the PEGMA hydrogel before and after freezing at $-20^{\circ} \mathrm{C}$. (b) Bending photographs of the PEGMA- $b$-PPGMA Janus copolymer strip (frozen at $-20^{\circ} \mathrm{C}$ for $24 \mathrm{~h}$ ) in DMF at $-20^{\circ} \mathrm{C}$.

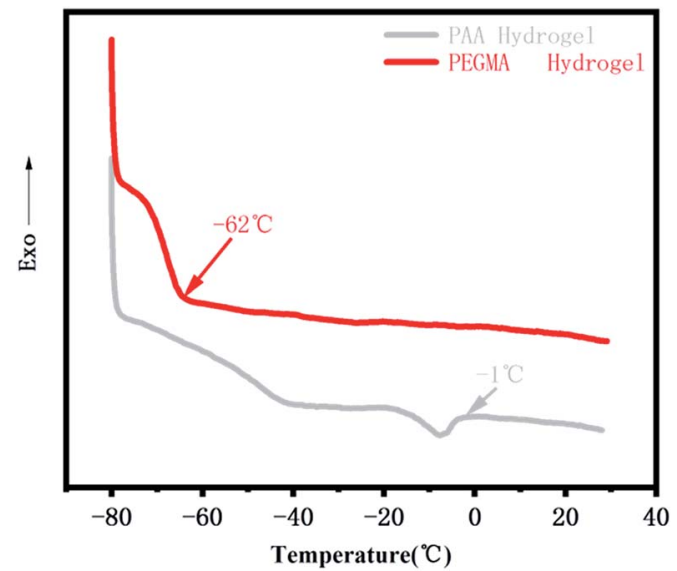

Fig. 7 Experimental DSC of the PAA hydrogel and the PEGMA hydrogel.

bent toward the PEGMA side, causing the left circuit to turn on and the left warning light was turned on (ESI Movie S3†).

By using the dual response characteristics of the PEGMA- $b$ PPGMA Janus copolymer, a smart device which provides a dual alarm and automatic disposal of solvent leaks can be designed, by fixing the Janus strip at the solvent leak line and placing two sensitive sensors above and below the Janus strip in the proper positions. When the amount of leaked solvent is within the allowable range, there is no reaction in the control room (Fig. S9a $\dagger$ ). When the amount of leaked solvent allows only less than half of the Janus strip to dissolve in the solvent, the lower

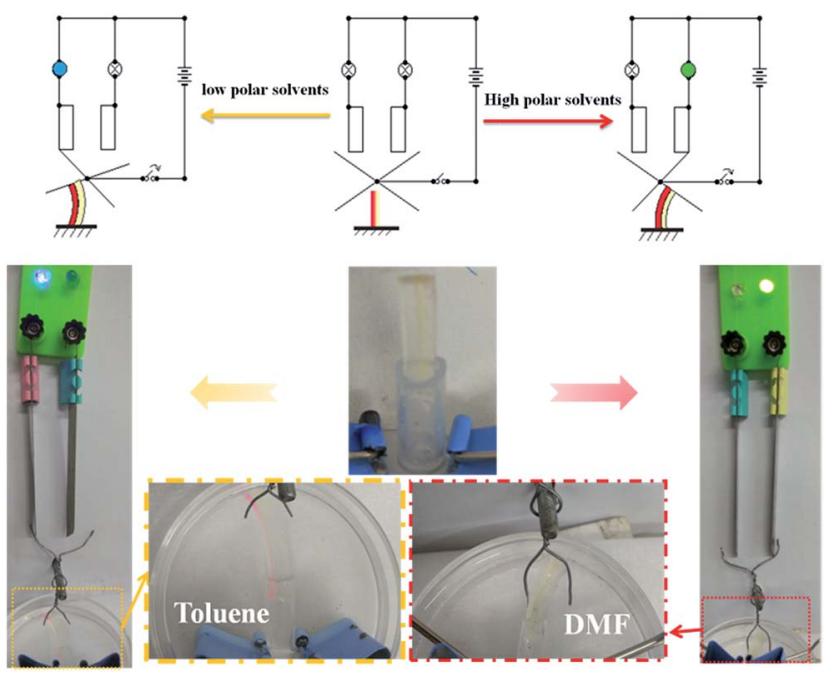

Fig. 8 Smart sensor for solvent leakage detection. A schematic representation of the smart sensor circuit (bottom). Bending of the PEGMA- $b$-PPGMA Janus strip in response to different solvents resulted in the turning-on of different electrodes. When organic solvents leaked, with toluene as an example, the strips bent towards the left with the PEGMA network facing inwards, including the electrode on the top of the strip. Then, the left circuit was connected, lighting the blue lamp on the left. Similarly, the green lamp on the right flashed when DMF was added, which is due to the strip bending towards the right with the PPGMA network facing inward, which closed the right circuit. 
part of the gel swells, causing the Janus strip to bend upwards, touching the upper sensor and making the right controller a green alarm (the control room turns green) which indicates that the amount of solvent leaking has reached a certain level and is close to the warning line (Fig. S9b $\dagger$ ). At this point, if no one is dealing with it, when the amount of leaked solvent completely exceeds the Janus strip, since the gel in the upper part begins to swell, the gel gradually flattens and bends downward, touching the sensor below. A red alarm is issued (the controller turns red) and the pump below starts to pump off the excess leaked solvent (Fig. S9c $\dagger$ ). As a result, it equips a dual alarm and automatic disposal function.

Due to the lack of mechanism in our material to dissipate significant amounts of mechanical energy under large deformation, the actuator cannot provide high mechanical force. To develop robust actuators, introducing physical interactions (such as host-guest interactions, hydrogen bonds, and dipoledipole interactions) into the present Janus actuator is our further research area to study. We believe that the designed material can provide new ideas for new materials in the fields of industry (automatic and safe collection of fractionation tower discharge), agriculture (automatic control of water volume), and medicine (early warning and automatic treatment of pleural effusion).

\section{Conclusion}

In summary, we successfully synthesized a bilayer and shape transition binary hydro/organo copolymer Janus strip by a onestep interfacial copolymerization. The binary synergistic PEGMA- $b$-PPGMA Janus strip, which is a synergistic interaction between the hydrophilic network and the hydrophobic network, showed unidirectional, bidirectional, and site-specific bending associated with solvent polarity through the synergistic swelling of the PEGMA network and the PPGMA network. Due to the hydrogen-bonded hydration between the PEGMA network and water in the PEGMA hydrogel, PEGMA hydrogels can still be flexible at low temperatures and show frost resistance, expanding the application range of the Janus actuator. The Janus strip's specific bending towards solvents allows it to be used as a smart sensor for solvent leaks and to determine solvent polarity. The dual response provided by the dual active layer provides the possibility of a double warning of solvent leaks. Our experimental results indicate that the amphiphilic polymer can also be used to develop hydro/organo actuators successfully by a one-step non-traditional interfacial polymerization. The performance of the hydro/organo actuators was improved accordingly and the practical application of the hydro/organo actuators was enlarged significantly. We believe that the design idea of actuators that can be used at low temperatures and that bend in relation to solvent polarity brings new approaches and ideas to new areas and applications.

\section{Conflicts of interest}

There are no conflicts to declare.

\section{Acknowledgements}

This work was supported by the National Natural Science Foundation of China (No. 51273189), the National Science and Technology Major Project of the Ministry of Science and Technology of China (No. 2016ZX05016), and the National Science and Technology Major Project of the Ministry of Science and Technology of China (No. 2016ZX05046).

\section{Notes and references}

1 S. Amador-Vargas, M. Dominguez, G. Leon, B. Maldonado, J. Murillo and G. L. Vides, Plant Ecology, 2014, 215, 14451454.

2 T. Vanden Driessche, Biol. Rhythm Res., 2000, 31, 451-468.

3 S. Armon, E. Efrati, R. Kupferman and E. Sharon, Science, 2011, 333, 1726-1730.

4 Y. Forterre, J. M. Skotheim, J. Dumais and L. Mahadevan, Nature, 2005, 433, 421-425.

5 M. J. Harrington, K. Razghandi, F. Ditsch, L. Guiducci, M. Rueggeberg, J. W. C. Dunlop, P. Fratzl, C. Neinhuis and I. Burgert, Nat. Commun., 2011, 2, 337.

6 T. F. Otero and J. M. Sansinena, Adv. Mater., 1998, 10, 491494.

7 Y. Osada, H. Okuzaki and H. Hori, Nature, 1992, 355, 242244.

8 C. S. Haines, M. D. Lima, N. Li, G. M. Spinks, J. Foroughi, J. D. W. Madden, S. H. Kim, S. Fang, M. J. de Andrade, F. Goktepe, O. Goktepe, S. M. Mirvakili, S. Naficy, X. Lepro, J. Oh, M. E. Kozlov, S. J. Kim, X. Xu, B. J. Swedlove, G. G. Wallace and R. H. Baughman, Science, 2014, 343, 868-872.

9 V. Magdanz, G. Stoychev, L. Ionov, S. Sanchez and O. G. Schmidt, Angew. Chem., Int. Ed., 2014, 53, 2673-2677.

10 F. Ilievski, A. D. Mazzeo, R. E. Shepherd, X. Chen and G. M. Whitesides, Angew. Chem., Int. Ed., 2011, 50, 18901895.

11 E. W. H. Jager, O. Inganas and I. Lundstrom, Science, 2000, 288, 2335-2338.

12 L. Zhang, H. Liang, J. Jacob and P. Naumov, Nat. Commun., 2015, 6, 7429.

13 J. Kim, J. A. Hanna, M. Byun, C. D. Santangelo and R. C. Hayward, Science, 2012, 335, 1201-1205.

14 M. J. N. Junk, W. Li, A. D. Schlueter, G. Wegner, H. W. Spiess, A. Zhang and D. Hinderberger, J. Am. Chem. Soc., 2011, 133, 10832-10838.

15 E. M. Ahmed, J. Adv. Res., 2015, 6, 105-121.

16 R. Longenecker, T. Mu, M. Hanna, N. A. D. Burke and H. D. H. Stoever, Macromolecules, 2011, 44, 8962-8971.

17 Y. Cao, N. Liu, C. Fu, K. Li, L. Tao, L. Feng and Y. Wei, ACS Appl. Mater. Interfaces, 2014, 6, 2026-2030.

18 Y. Pan, Y. Gao, J. Shi, L. Wang and B. Xu, J. Mater. Chem., 2011, 21, 6804-6806.

19 K. Okeyoshi and R. Yoshida, Soft Matter, 2009, 5, 4118-4123.

20 K. Shi, Z. Liu, Y.-Y. Wei, W. Wang, X.-J. Ju, R. Xie and L.-Y. Chu, ACS Appl. Mater. Interfaces, 2015, 7, 27289-27298. 
21 W. Jiang, D. Niu, H. Liu, C. Wang, T. Zhao, L. Yin, Y. Shi, B. Chen, Y. Ding and B. Lu, Adv. Funct. Mater., 2014, 24, 7598-7604.

22 R. Luo, J. Wu, N.-D. Dinh and C.-H. Chen, Adv. Funct. Mater., 2015, 25, 7272-7279.

23 B.-y. Lee, S. Hyun, G. Jeon, E. Y. Kim, J. Kim, W. J. Kim and J. R. Kim, ACS Appl. Mater. Interfaces, 2016, 8, 11758-11764.

24 X. M. Liu, L. S. Wang, L. Wang, J. C. Huang and C. B. He, Biomaterials, 2004, 25, 5659-5666.

25 G. S. Longo, M. O. de la Cruz and I. Szleifer, Soft Matter, 2016, 12, 8359-8366.

26 S. Zakharchenko and L. Ionov, ACS Appl. Mater. Interfaces, 2015, 7, 12367-12372.

27 G. Stoychev, N. Puretskiy and L. Ionov, Soft Matter, 2011, 7, 3277-3279.

28 L. Ionov, Adv. Funct. Mater., 2013, 23, 4555-4570.

29 F. Zhang, J. Fan, P. Zhang, M. Liu, J. Meng, L. Jiang and S. Wang, NPG Asia Mater., 2017, 9, e380.

30 X. P. Morelle, W. R. Illeperuma, K. Tian, R. Bai, Z. Suo and J. J. Vlassak, Adv. Mater., 2018, e1801541.

31 I. Yati, K. Karadag and H. B. Sonmez, Polym. Adv. Technol., 2015, 26, 635-644.

32 B. Ivan, J. P. Kennedy and P. W. Mackey, ACS Symp. Ser., 1991, 469, 194-202.

33 B. Ivan, J. P. Kennedy and P. W. Mackey, ACS Symp. Ser., 1991, 469, 203-212.

34 C. Lv, H. Xia, Q. Shi, G. Wang, Y.-S. Wang, Q.-D. Chen, Y.-L. Zhang, L.-Q. Liu and H.-B. Sun, Adv. Mater. Interfaces, 2017, 4, 1601002.

35 D. Dikovsky, H. Bianco-Peled and D. Seliktar, Biomaterials, 2006, 27, 1496-1506.
36 N. C. Padmavathi and P. R. Chatterji, Macromolecules, 1996, 29, 1976-1979.

37 Y. T. Demirel, I. Yati, R. Donmez and H. B. Sonmez, Chem. Eng. J., 2017, 312, 126-135.

38 R. D. Rogers and J. H. Zhang, J. Chromatogr. B: Biomed. Sci. Appl., 1996, 680, 231-236.

39 S. J. Kim, K. J. Lee, I. Y. Kim, K. H. An and S. I. Kim, J. Appl. Polym. Sci., 2003, 90, 1384-1388.

40 P. H. Ni, Q. S. Pan, L. S. Zha, C. C. Wang, A. Elaissari and S. K. Fu, J. Polym. Sci., Part A-1: Polym. Chem., 2002, 40, 624-631.

41 E. A. Yapar and O. Inal, Trop. J. Pharm. Res., 2012, 11, 855866.

42 S. Shukla and J. S. P. Rai, Int. J. Plast. Technol., 2013, 17, 182193.

43 G. Malucelli, G. Gozzelino, F. Ferrero, R. Bongiovanni and A. Priola, J. Appl. Polym. Sci., 1997, 65, 491-497.

44 H. Jia, Z. Huang, Z. Fei, P. J. Dyson, Z. Zheng and X. Wang, J. Mater. Chem. B, 2017, 5, 8193-8199.

45 Y. Cheng, K. Ren, D. Yang and J. Wei, Sens. Actuators, B, 2018, 255, 3117-3126.

46 T. Wu, J. Li, J. Li, S. Ye, J. Wei and J. Guo, J. Mater. Chem. C, 2016, 4, 9687-9696.

47 T. Hauet and M. Eugene, Kidney Int., 2008, 74, 998-1003.

48 M. A. Bag and L. M. Valenzuela, Int. J. Mol. Sci., 2017, 18, 1422.

49 H. Kitano, K. Ichikawa, I. Ide, M. Fukuda and W. Mizuno, Langmuir, 2001, 17, 1889-1895.

50 F. Gnanaprakasam Thankam and J. Muthu, RSC Adv., 2013, 3, 24509-24520.

51 G. T. Finosh and M. Jayabalan, $R S C A d v .$, 2015, 5, 3818338201. 